\title{
Asymmetric dust distribution in an eccentric protoplanetary disk as a signpost of a gas giant planet
}

\author{
Pin-Gao Gu, He-Feng Hsieh and Hsi-Wei Yeh \\ Instituter of Astronomy \& Astrophysics, Academia Sinica, \\ Taipei 10617, Taiwan \\ email: gu@asiaa.sinica.edu.tw
}

\begin{abstract}
The presence of a cavity in a protoplanetary disk revealed by dust continuum emissions is sometimes postulated as a signpost of an embedded gas giant planet. More peculiarly, dust emissions exterior to the cavity are often observed to be asymmetric. We explore the possibility of the asymmetry as a result of the asymmetric distribution of dust in an eccentric protoplanetary disk under the secular gravitational perturbation of an embedded massive gas giant planet. We find that the surface density of the dust well coupled to the disk gas is enhanced around the apocenter of the disk. In addition, the azimuthal distributions of particles of various sizes can deviate significantly due to different coupling to the gas. Overall, the asymmetric structure exhibits a phase correlation between the gas velocity field and dust density distribution. A Doppler map for an eccentric disk is also presented based on Cycle 1 ALMA observations. Our study potentially provides a reality check as to whether an asymmetric disk gap detected at sub-mm and $\mathrm{cm}$ wavelengths is a signpost of a massive gas giant planet.
\end{abstract}

\section{Introduction}

The presence of a cavity and/or non-axisymmetric features in a protoplanetary disk as revealed by dust continuum emissions has at times been postulated as a potential signpost of an embedded gas giant planet (Andrews et al. 2011). If the planet is sufficiently massive, the disk exterior to the planet's orbit can become moderately eccentric (thus we only show the results for the outer disk. See below) and start precessing on the secular timescale, depending on the disk mass and viscosity (Dunhill et al. 2012). In such an eccentric protoplanetary disk harboring a massive planet, the orbits of dust particles would be eccentric as well due to the gas drag.

We run the FARGO code (Masset 2000) to obtain the gas profile in a two-dimensional disk with a massive planet, and then employ the secular perturbation theory and incorporate aerodynamic drag (Paardekooper et al. 2008) to estimate the dust velocity and density that will in turn reveal the asymmetric structures inherent to an eccentric disk. The purpose of this study is to focus only on the secular behaviors of dust associated with an eccentric protoplanetary disk in the presence of a massive giant planet. It should be noted that although the vortex model produces a much larger dust density contrast than that in our model (van der Marel et al. 2013), the disk conditions required for vortex generation in general allow for the generation of disk eccentricity.

\section{Results}

Fig. 1 shows a snapshot of the spatial distribution of the dust surface density for $0.01 \mathrm{~cm}$ and $1 \mathrm{~m}$ particles in both the protoplanetary and anemic (low-density transitional) disks. The distributions exhibit $m=1$ structures with the density excess about 


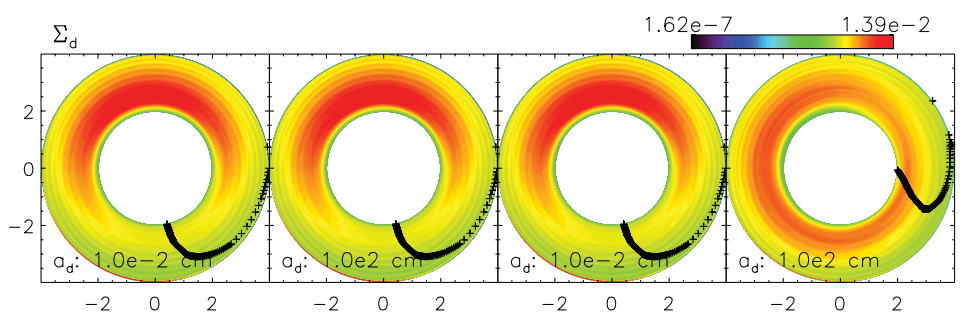

Figure 1. Spatial distribution of the dust surface density for $0.01 \mathrm{~cm}$ and $1 \mathrm{~m}$ particles in the cases of the protoplanetary (left 2 panels) and anemic disks (right 2 panels). The units of the surface density are about $0.356 \mathrm{~g} / \mathrm{cm}^{2}$ and $9 \times 10^{-4} \mathrm{~g} / \mathrm{cm}^{2}$ for the protoplanetary and anemic disks, respectively. The plus signs in the plot mark the azimuthally averaged longitude of pericenter of particles.

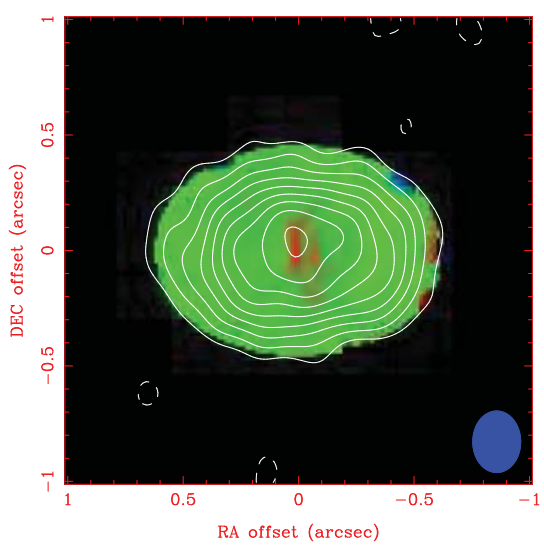

Figure 2. Doppler image of an eccentric disk. Green denotes the Keplerian circular rotation. Red indicates an excess of redshifted velocities elongated along the minor axis of the disk.

$5-10 \%$ around the apocenter. The dust enhancement is caused by the gas streamlines converge near the apocenter of the disk. All the $m=1$ dust distributions look quite similar except for that for the $1 \mathrm{~m}$ particles in the anemic disk shown in the lower bottom panel. For those large particles in the anemic disk, the dimensionless secular timescale $\tau_{s, s e c} \approx$ 1-10 and thus the particles are weakly coupled. As a result, the $1 \mathrm{~m}$ particles precess with the gas disk but with a phase lag $\approx \arctan \tau_{s, s e c} \approx 80^{\circ}$, as illustrated in Fig. 1 .

Fig. 2 presents a Doppler image of the simulated protoplanetary disk subtracted from the Keplerian circular motion (colors), overlaid on the total integrated intensity map at $345.8 \mathrm{GHz}$ corresponding to ${ }^{12} \mathrm{CO} 3-2$ line (contours). A planet of $5 M_{j}$ is placed at 20 AU on the disk plane. The disk has the radius of $80 \mathrm{AU}$ at $140 \mathrm{pc}$ away and is inclined by 45 degrees from the line of sight. This simulated observational map is constructed using Cycle 1 ALMA observations with 6 hour integration, an $0.27^{\prime \prime} \times 0.2^{\prime \prime}$ angular resolution, and an $0.11 \mathrm{~km} / \mathrm{s}$ velocity resolution.

\section{References}

Andrews, S. M., et al. 2011, ApJ, 732, 42

Dunhill, A., Alexander, R., \& Armitage, P. 2013, MNRAS, 428, 3072

Masset, F. 2000, A\&AS, 141, 165

Paardekooper, S.-J., Thébault, P., \& Mellema, G. 2008, MNRAS, 386, 973

van der Marel, N., et al. 2013, Science, 340, 1199 